\title{
Anomalies in a waterlike model confined between plates
}

Cite as: J. Chem. Phys. 138, 084505 (2013); https://doi.org/10.1063/1.4792639

Submitted: 19 November 2012 . Accepted: 04 February 2013. Published Online: 25 February 2013

Leandro B. Krott, and Marcia C. Barbosa
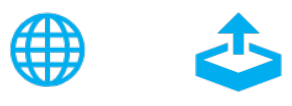

\section{ARTICLES YOU MAY BE INTERESTED IN}

A general purpose model for the condensed phases of water: TIP4P/2005

The Journal of Chemical Physics 123, 234505 (2005); https://doi.org/10.1063/1.2121687

Effects of confinement on anomalies and phase transitions of core-softened fluids

The Journal of Chemical Physics 142, 134502 (2015); https://doi.org/10.1063/1.4916563

Confinement effects on the liquid-liquid phase transition and anomalous properties of a monatomic water-like liquid

The Journal of Chemical Physics 143, 244503 (2015); https://doi.org/10.1063/1.4937486

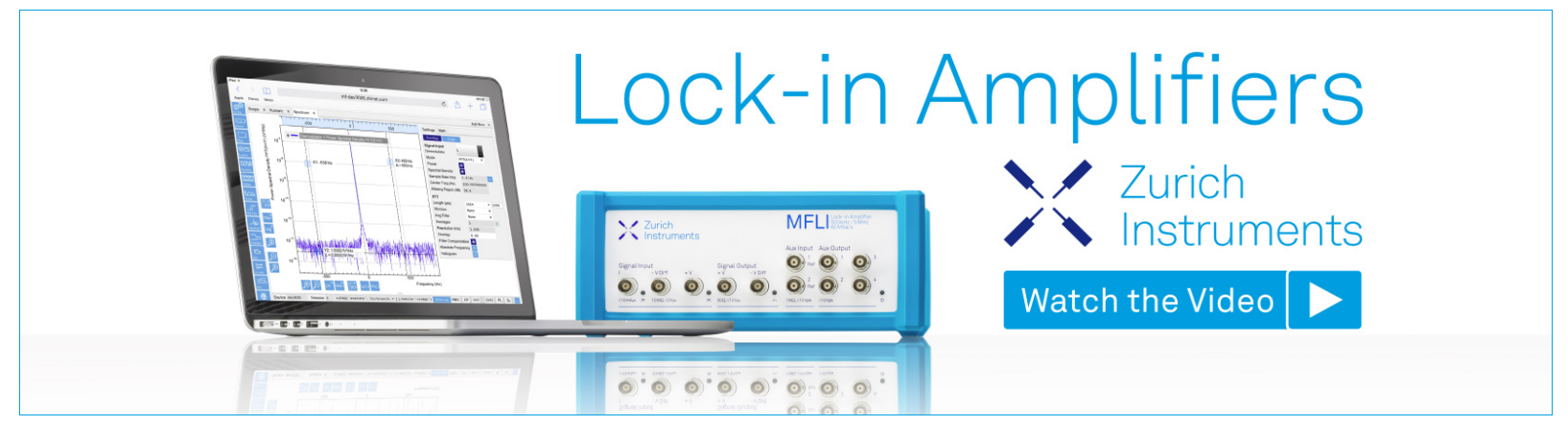




\title{
Anomalies in a waterlike model confined between plates
}

\author{
Leandro B. Krott and Marcia C. Barbosa \\ Instituto de Física, Universidade Federal do Rio Grande do Sul, 91501-970 Porto Alegre, \\ Rio Grande do Sul, Brazil
}

(Received 19 November 2012; accepted 4 February 2013; published online 25 February 2013)

\begin{abstract}
Using molecular dynamic simulations we study a waterlike model confined between two fixed hydrophobic plates. The system is tested for density, diffusion, and structural anomalous behavior and compared with the bulk results. Within the range of confining distances we had explored and observed that in the pressure-temperature phase diagram the temperature of maximum density (TMD line) and the temperature of maximum and minimum diffusion occur at lower temperatures when compared with the bulk values. For distances between the two layers below a certain threshold, $d \leq d_{c}$, only two layers of particles are formed, for $d \geq d_{c}$ three or more layers are formed. In the case of three layers the central layer stays liquid while the contact layers crystallize. This result is in agreement with simulations for atomistic models. (C) 2013 American Institute of Physics. [http://dx.doi.org/10.1063/1.4792639]
\end{abstract}

\section{INTRODUCTION}

Water has several peculiar thermodynamic and dynamic properties not observed in other liquids. This is the case of the density at room pressure that has a maximum at $4{ }^{\circ} \mathrm{C}^{1-3}$ while in most materials the density increases monotonically with the decrease of the temperature. In addition, between $0.1 \mathrm{MPa}$ and $190 \mathrm{MPa}$ water also exhibits an anomalous increase of compressibility ${ }^{4,5}$ and, at atmospheric pressure, an increase of isobaric heat capacity upon cooling. ${ }^{6,7}$ Besides the thermodynamic anomalies water also exhibits an unusual behavior in its mobility. The diffusion coefficient for normal liquids increases with the decrease of pressure, for water it has a maximum at $4{ }^{\circ} \mathrm{C}$ for $1.5 \mathrm{~atm} .{ }^{3,8}$

The presence of the large increase in the response function induced the idea of the existence of two liquid phases and a critical point. ${ }^{9}$ The possibility of the existence of the second critical point has been supported by extensive simulations. ${ }^{10-12}$ This liquid-liquid coexistence is located at the supercooled region beyond the line of homogeneous nucleation and thus cannot be experimentally measured. In order to circumvent this inconvenience, experiments in confined water were performed. ${ }^{13,14}$ They showed that the large increase of the specific heat is actually a peak that can be associated with the Widom line. This line is found ${ }^{14}$ as the continuation of the liquid-liquid coexistence line beyond the critical point at the one phase region. ${ }^{13,14}$

The drawback of experiments in nanoscale confinement is that the results obtained do not necessarily lead to conclusions at the bulk level. Notwithstanding this disadvantage the study of confined water by itself is interesting since water is present in ionic channels, proteins, vesicles, and other cellular structures under nanoscale confinement. In order to understand the behavior of water under these limitations a number of experiments and simulations of confined water have been performed.

Several types of confinement have been explored: experiments in cylindrical porous ${ }^{15-17}$ and simulations in carbon nanotubes, ${ }^{18-21}$ simulations in one pore, ${ }^{22-26}$ experiments in one pore, ${ }^{13,27,28}$ simulations in porous matrices, ${ }^{29-32}$ experiments $\mathrm{s}^{33,34}$ and simulations in rough surfaces ${ }^{35-40}$ and simulations in flat plates. ${ }^{36,38,41,42}$

In particular, $\mathrm{x}$-ray and neutron scattering with water in nanopores show that the liquid state persists down to temperature much lower than in bulk. ${ }^{15,43,44}$ In these experiments in the case of hydrophobic walls the liquid-crystal transition occurs at lower temperatures than in the case of hydrophilic walls. ${ }^{28,43}$ In some scattering experiments there are indications of the formation of cubic ice instead of the hexagonal ice present in the bulk. ${ }^{16,45}$ Several of these experiments show evidences of the presence of layers, one close to the walls and one at the center. ${ }^{15,16,43,44}$ For certain type of walls, the central crystallizes before the wall layers. ${ }^{15}, 16$ Therefore, the experimental results are not conclusive. They indicate that the crystallization in confined water depends strongly on the size of the porous ${ }^{15,17,44,46,47}$ and on the level of hydration water under surfaces. ${ }^{33,45}$

However, diffraction studies give only indirect information about the existence of crystalline or amorphous states in water, because the Bragg peaks of ice are quite hard to distinguish from liquid states. Moreover, the presence of layers is also only obtained from indirect evidences. In order to circumvent the difficulties of obtaining the structure of water inside the confined system from experiments, a number of simulations have been performed. ${ }^{36,48,49}$ They employ atomistic models such as SPC/E $\mathrm{E}^{22-24,48-50}$ and TIP5P $\mathrm{P}^{36}$ and coarsegrained models ${ }^{29,51}$ for water.

Simulations indicate that for both hydrophobic ${ }^{36,42,48-50}$ and hydrophilic ${ }^{48-50}$ surfaces two or three layers are formed depending on the distance between the confining surfaces. ${ }^{24}$ In the case of hydrophobic walls, there is a phase transition between the two to the three layers regime and for a certain temperature and layer separation the central layer stays liquid while the molecules at the walls crystallize. In addition, in the case of hydrophobic walls the temperature of maximum 
density and the temperature of maximum and minimum diffusivity move to lower temperatures when compared with the bulk results. ${ }^{36,42}$ At very low pressures, cavitation appears. ${ }^{50}$ In the case of the hydrophilic walls, in agreement with the experimental results, the system remains liquid for temperatures below the temperatures in the bulk case. ${ }^{48}$

Thermodynamic anomalies do not occur only in water, experiments for $\mathrm{Te},{ }^{52} \mathrm{Ga}, \mathrm{Bi}, \mathrm{S},{ }^{53,54}$ and $\mathrm{Ge}_{15} \mathrm{Te}_{85},{ }^{55}$ liquid metals, ${ }^{56}$ and graphite, ${ }^{57}$ and simulations for silica, ${ }^{58-60}$ silicon, ${ }^{61}$ and $\mathrm{BeF}_{2}{ }^{58}$ shown that these system also have thermodynamic anomalies. In addition, silica ${ }^{60,62}$ and silicon ${ }^{63}$ show diffusion anomalous behavior. In principle these systems under confinement could also show a shift in the anomalous properties and layering without having hydrogen bonds. Atomistic and coarse-grained models ${ }^{29,51}$ for water are an interesting tool for understanding water and its properties, however they are not appropriated for seeking for universal mechanisms that would be common for water and the materials cited above in which the hydrogen bonds are not present but still they present the anomalous behavior of water.

Acknowledging that core softened (CS) potentials may engender density and diffusion anomalous behavior, a number of CS potentials were proposed to model the anisotropic systems described above. They possess a repulsive core that exhibits a region of softening where the slope changes dramatically. This region can be a shoulder or a ramp. ${ }^{14,64-89}$ Despite their simplicity, such models had successfully reproduced the thermodynamic, dynamic, and structural anomalous behavior present in bulk liquid water. They also predict the existence of a second critical point hypothesized by Poole and coworkers. ${ }^{9}$ This suggests that some of the unusual properties observed in water can be quite universal and possibly present in other systems.

In this work we study the effect of the confinement in particles interacting through a CS potential. Our model for the fluid is a potential ${ }^{78,79}$ with hard core followed by a smooth shoulder and a tiny attractive region. The absence of the deep attractive well simplifies the system so it does not have critical regions. In addition it does not have any directionality and therefore it is not water. However, it does exhibit the density, the diffusion, and the response functions anomalies observed in water. This suggests that some of the anomalous properties that are attributed directionality of water can be found in spherical symmetry systems.

We explore that also some of the properties of water under confinement such as the presence of layering and the shift to lower temperatures of maximum density and of maximum and minimum of the diffusion coefficient can also be obtained with CS potentials.

The paper is organized as follows: in Sec. II we introduce the model; in Sec. III the methods and simulation details are described; the results are given in Sec. IV; and finally, the conclusions in Sec. V.

\section{THE MODEL}

We study a system of $N$ particles with diameter $\sigma_{p}$ confined between two fixed plates. The surfaces are formed by particles with diameter $\sigma_{w}$ which are organized in a square

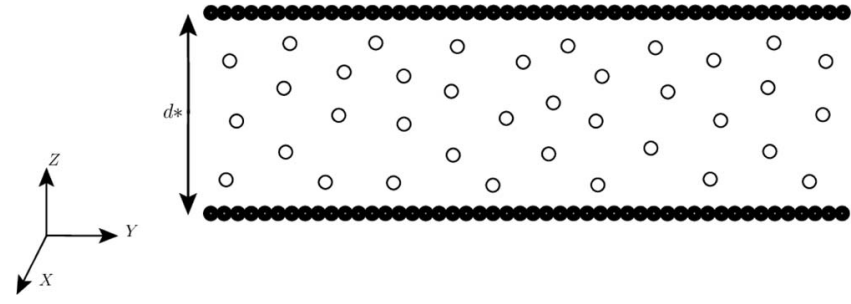

FIG. 1. Model system of particles confined between plates.

lattice of area $L^{2}$. The center-to-center plates distance is $d^{*}=d / \sigma_{p}$. A schematic depiction of the system is shown in Fig. 1.

The particles confined between the two plates interact through an isotropic effective potential (Fig. 2) given by

$$
\frac{U(r)}{\epsilon}=4\left[\left(\frac{\sigma_{p}}{r}\right)^{12}-\left(\frac{\sigma_{p}}{r}\right)^{6}\right]+a \exp \left[-\frac{1}{c^{2}}\left(\frac{r-r_{0}}{\sigma_{p}}\right)^{2}\right] .
$$

The first term is a standard Lennard-Jones (LJ) 12-6 potential with $\epsilon$ depth plus a Gaussian centered on radius $r=$ $r_{0}$ and width $c$. We used parameters $a=5, r_{0} / \sigma_{p}=0.7$, and $c=1$. The pressure versus temperature phase diagram of this system in the bulk was studied by Oliveira et $a l^{78,79}$ They found that a system or particles interacting through this potential exhibits a region in the pressure-temperature phase diagram where the density and diffusion coefficient are anomalous.

This potential has two length scales with a repulsive shoulder at $r / \sigma_{p} \approx 1$ and a very small attractive well at $r / \sigma_{p}$ $\approx 3.8$ (Fig. 2). Depending of the choice of the parameters $a$, $b, c$, and $\sigma_{p}$, it can represent a whole family of intermolecular interactions. In this paper we employ $a=5, r_{0} / \sigma_{p}=0.7$, and $c=1$.

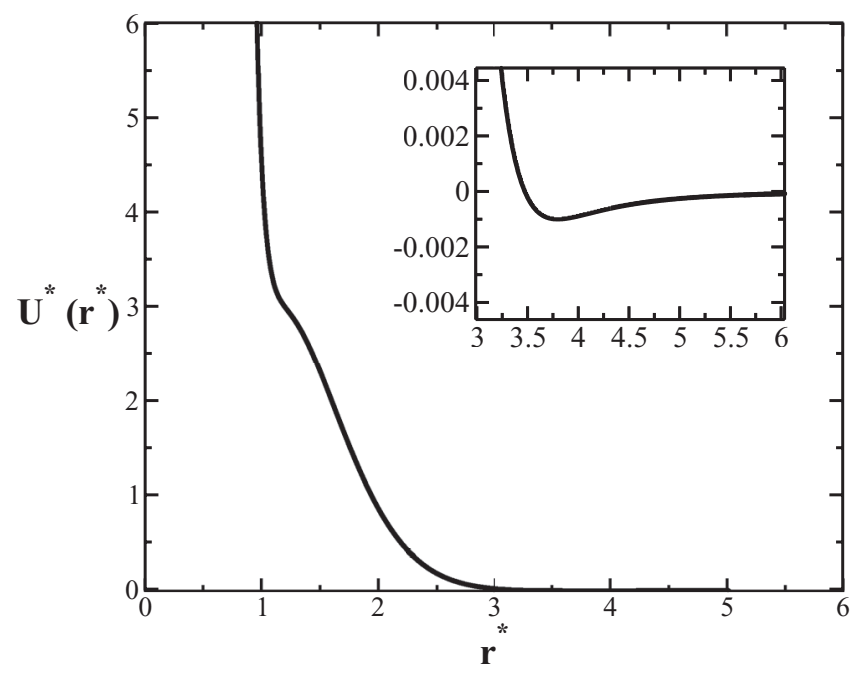

FIG. 2. Isotropic effective potential equation (1) of interaction between the waterlike particles. The potential and the distances are in dimensionless units, $U^{*}=U / \epsilon$ and $r^{*}=r / \sigma_{p}$ and the parameters are $a=5, r_{0} / \sigma_{p}=0.7$, and $c=1$. The inset shows a zoom in the very small attractive part of the potential. 
The particle-plate interaction is given by WeeksChandler-Andersen Lennard-Jones potential, namely, ${ }^{90,91}$

$$
U=\left\{\begin{array}{l}
U_{\mathrm{LJ}}(r)-U_{\mathrm{LJ}}\left(r_{c w}\right), \quad r \leq r_{c w}, \\
0, \quad r>r_{c w},
\end{array}\right.
$$

where $U_{L J}(r)$ is a standard 12-6 LJ potential. The cutoff distance is $r_{c w}=2^{1 / 6} \sigma_{w p}$, where $\sigma_{w p}=\left(\sigma_{p}+\sigma_{w}\right) / 2$ is the Lorentz-Berthelot mixing rule ${ }^{92}$ used when two kinds of particles are interacting between them. In our model, $\sigma_{p}=\sigma_{w}$ $=\sigma_{w p}$.

\section{THE METHODS AND SIMULATION DETAILS}

The system has 507 particles confined between the plates with area $L^{2}$ and distant $d$, resulting in a number density $\rho$ $=N /\left(d L^{2}\right)$. The plates are located at $z=0$ and $z=d$, whereas in $x$ and $y$ directions periodic boundary conditions are used. The repulsive interactions with the plates underestimate the number density, so we need to calculate the effective density using the effective distance $d_{e}$ perpendicular to the plates. The new density will be $\rho=N /\left(d_{e} L^{2}\right)$, where $d_{e} \approx d-\left(\sigma_{p}+\sigma_{w}\right) / 2$ is an approach for the effective distance between the plates. ${ }^{36}$

Molecular dynamics simulations at the NVT-constant ensemble and the Nose-Hoover ${ }^{93,94}$ thermostat were used in order to keep fixed the temperature, with coupling parameter $Q=2$. The interaction potential between particles has a cutoff of $r_{c}=3.5$ and this potential was shifted in order to have $U=0$ at $r_{c}$.

Several densities and temperatures are calculated for the following distances $d^{*}=d / \sigma_{p}$ between the plates: 4.2, 4.8, 5.5, 6.0, and 6.3. The initial configurations of the systems were set on solid structure and the equilibrium states reached after $2 \times 10^{6}$ steps, followed by $4 \times 10^{6}$ simulation run. The time step was 0.002 in reduced units and the average of the physical quantities was obtained with 50 decorrelated samples. The thermodynamic stability of the system was checked by analyzing the dependence of parallel and perpendicular pressure on density, namely, and by the behavior of the energy after the equilibrium states.

The thermodynamic averages in parallel and perpendicular directions to the plates are calculated employing different procedures. ${ }^{95}$ Parallel pressure, $P_{\|}$, is computed using the Virial expression for the $x$ and $y$ directions, ${ }^{36,41,42,96}$ while the perpendicular pressure, $P_{\perp}$, is calculated using two distinct methods. For systems with a strong confinement, such as $d^{*}=4.2$ and 4.8 , the total force perpendicular to the plates is used, ${ }^{41,97}$

$$
P_{\perp}=\frac{F_{\text {plates }}}{A}=\frac{\left|\sum_{i=1}^{N} \mathbf{F}_{i, \text { plates }}\right|}{L^{2}} .
$$

For the others systems with larger distances, such as $d^{*}=5.5$, 6.0 , and 6.3 , the pressure $P_{\perp}$ is computed through the Virial expression in $z$ direction. ${ }^{98}$ In this framework the Helmholtz free energy is analyzed in terms of area, $A=L_{x} L_{y}$, distance between the plates, $L_{z}$. Thanks to the periodic boundary condition in the plane, the system is extensive in the area but not in the distance between the plates. Therefore, only the parallel pressure can be regarded as a thermodynamic quantity and it might scale as the experimental pressure.

The dynamic of the systems was studied by lateral diffusion coefficient, $D_{\|}$, related with the mean square displacement (MSD) from Einstein relation,

$$
D_{\|}=\lim _{\tau \rightarrow \infty} \frac{\left\langle\Delta r_{\|}(\tau)^{2}\right\rangle}{4 \tau},
$$

where $r_{\|}=\left(x^{2}+y^{2}\right)^{1 / 2}$ is the distance between the particles parallel to the plates.

We also studied the structure of the systems by lateral radial distribution function, $g_{\|}\left(r_{\|}\right)$, and translational order parameter, $t$. We calculate the $g_{\|}\left(r_{\|}\right)$in specific regions between the plates, and the same for parameter $t$. An usual definition for $g_{\|}\left(r_{\|}\right)$is

$$
g_{\|}\left(r_{\|}\right) \equiv \frac{1}{\rho^{2} V} \sum_{i \neq j} \delta\left(r-r_{i j}\right)\left[\theta\left(\left|z_{i}-z_{j}\right|\right)-\theta\left(\left|z_{i}-z_{j}\right|-\delta z\right)\right]
$$

The $\theta(x)$ is the Heaviside function and it restricts the sum of particle pairs in the same slab of thickness $\delta z=1$. We need to compute the number of particles for each region and the normalization volume will be cylindrical. The $g_{\|}\left(r_{\|}\right)$is proportional to the probability of finding a particle at a distance $r_{\|}$from a referent particle.

The translational order parameter is defined as ${ }^{60,99,100}$

$$
t \equiv \int_{0}^{\xi_{c}}\left|g_{\|}(\xi)-1\right| d \xi
$$

where $\xi=r_{\|} \rho_{S}^{1 / 2}$ is the interparticle distance in the direction parallel to the plates scaled by $\rho_{s}^{1 / 2}=\left(N_{\text {layer }} / L^{2}\right)^{1 / 2} \cdot N_{\text {layer }}$ is the average of particles for each slab supposing that this number does not change significantly (well-defined layers). ${ }^{98}$ We use $\xi_{c}=\rho_{s}^{1 / 2} L / 2$ as cutoff distance.

When the system is an ideal gas, with $g_{\|}\left(r_{\|}\right)=1$, we obtain $t=0$, because the system is not structured. But, as the system becomes more structured, like a crystal phase, the $g_{\|}\left(r_{\|}\right) \neq 1$, so parameter $t$ assumes large values.

All physical quantities are shown in reduced units ${ }^{92}$ as

$$
\begin{aligned}
d^{*} & =\frac{d}{\sigma_{p}}, \\
\tau^{*} & =\frac{(\varepsilon / m)^{1 / 2}}{\sigma_{p}} \tau, \\
T^{*} & =\frac{k_{B}}{\varepsilon} T, \\
P_{\|, \perp}^{*} & =\frac{\sigma_{p}^{3}}{\varepsilon} P_{\|, \perp}, \\
\rho^{*} & =\sigma_{p}^{3} \rho, \\
D_{\|}^{*} & =\frac{(m / \varepsilon)^{1 / 2}}{\sigma_{p}} D_{\|} .
\end{aligned}
$$

\section{RESULTS}

\section{A. Systems with three layers}

The first set of systems that we study corresponds to plates separated by the distances $d^{*}=5.5,6.0$, and 6.3. In 
(a)

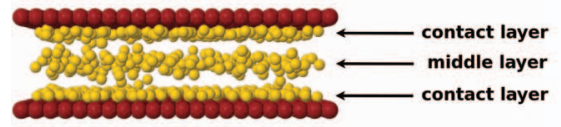

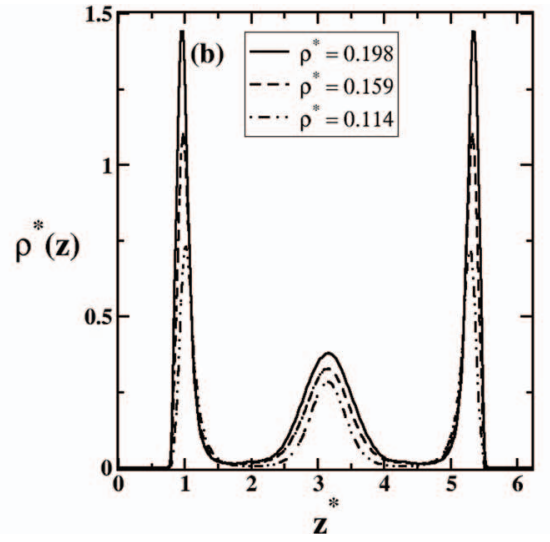

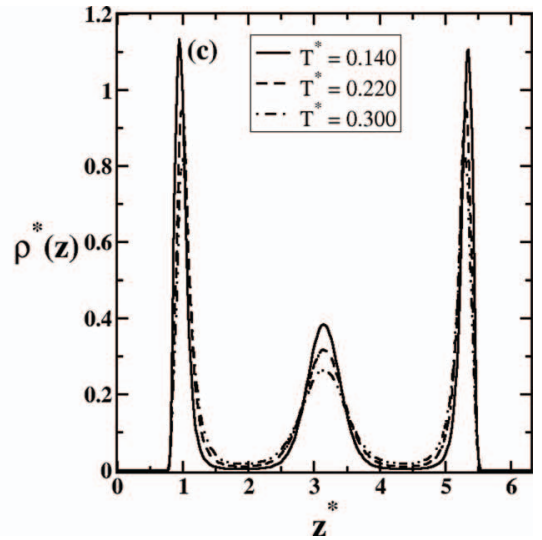

FIG. 3. In (a), we have a snapshot of the system with $T^{*}=0.220$ and $\rho^{*}=0.141$ after the equilibrium state. Furthermore, the transversal density profile is shown for (b) $T^{*}=0.220$ and different densities, and for (c) $\rho^{*}=0.141$ and different temperatures. We can see the formation of two contact layers, near the plates, and one middle layer. This system corresponds to $d^{*}=6.3$, whereas the cases such as $d^{*}=5.5$ and 6.0 present the same behavior in relation to layering density.

all these cases, the particles are structured in three layers in $z$ direction divided in two contact layers, near the plates, and one middle layer, located in the center of the plates. The formation of layering structures in confined water was also observed in atomistic models. ${ }^{36,42}$ The layering density can be seen in Fig. 3 that illustrates the $d^{*}=6.3$ case: (a) the snapshot of the system with $T^{*}=0.220$ and $\rho^{*}=0.141$, (b) the transversal density profile for $T^{*}=0.220$ and various densities, and (c) the transversal density profile for $\rho^{*}$ $=0.141$ and different temperatures. The layers become more defined at low temperatures and high densities. Now we need to identify if the different layers are in the solid or in the liquid state. In order to answer to this question the structure is analyzed.

Figure 4 shows the radial distribution function for $d^{*}=6.3$ in two cases: (a) $\rho^{*}=0.181$ and $T^{*}=0.220$ and (b) $\rho^{*}=0.217$ and $T^{*}=0.140$. For the case (a) the radial distribution of the central layer and of the contact layer is liquidlike. The contact layer shows a distribution compatible with a very structured liquid. For the case (b), the central layer is also liquidlike, however the contact layer is solidlike. The liquid- solid transition occurs at different temperatures and densities for the confinements $d^{*}=5.5,6.0$ and $d^{*}=6.3$ that do exhibit the three layers analyzed here. This result is in agreement with observations for SPC/E water. ${ }^{42}$

In the case of the bulk ${ }^{79}$ the potential exhibits an anomalous behavior in the translational order parameter $t^{*}$. For normal systems the $t^{*}$ grows with the density, however, for our CS potential it has a region where it does decreases with the increase of the density. Here, we test if this anomalous behavior is also observed in the central and contact layers.

Figure 5(a) and 5(b) show the translational order parameter defined by Eq. (6) as function of density for different temperatures $T^{*}=0.170,0.190,0.205,0.220,0.232,0.245$, 0.260 , and 0.300 , at $d^{*}=6.3$, for the contact layer and for the middle layer, respectively. The dots represent the simulation data and the solid lines identify the isotherms. The dashed lines, $\rho_{t-\max }^{*}<\rho^{*}<\rho_{t-\min }^{*}$, identifies the region in which $t^{*}$ is anomalous, namely, it decreases with the increase of the density. The region in the pressure-temperature phase diagram in which $t^{*}$ is computed under confinement occurs at lower temperatures when compared with the bulk values. ${ }^{79}$
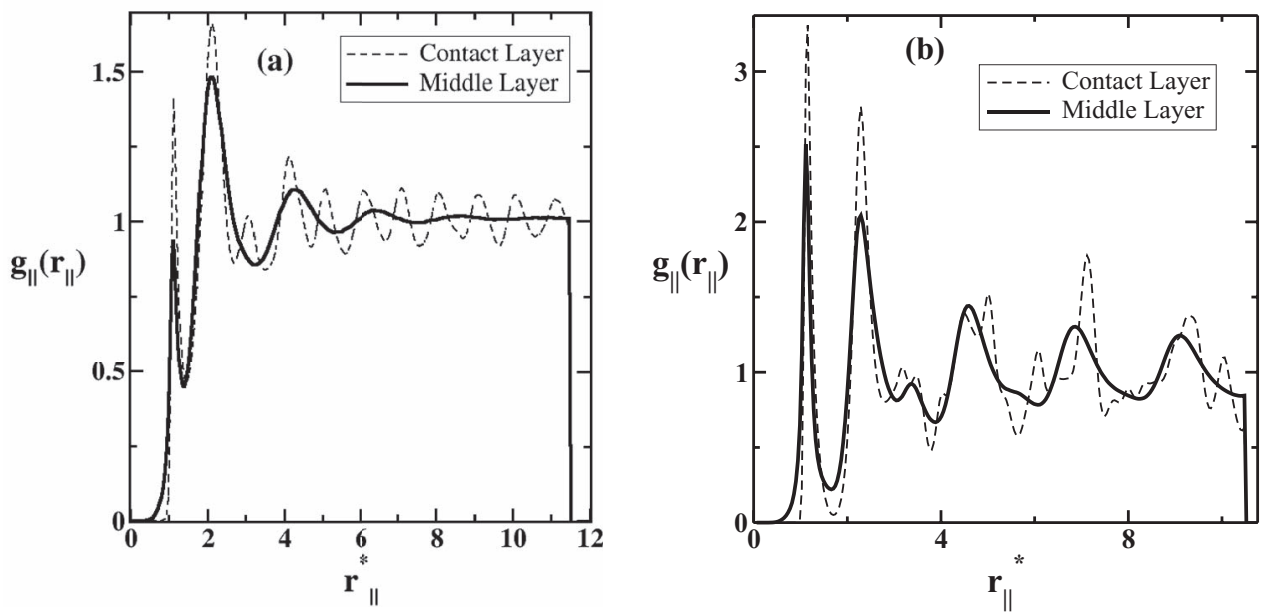

FIG. 4. Radial distribution function for $\rho^{*}=0.181$ and $T^{*}=0.220$ in (a), and $\rho^{*}=0.217$ and $T^{*}=0.140$ in (b). Bold lines represent the $g_{\|}\left(r_{\|}\right)$for the middle layer and the dashed lines represent the $g_{\|}\left(r_{\|}\right)$for the contact layer. 

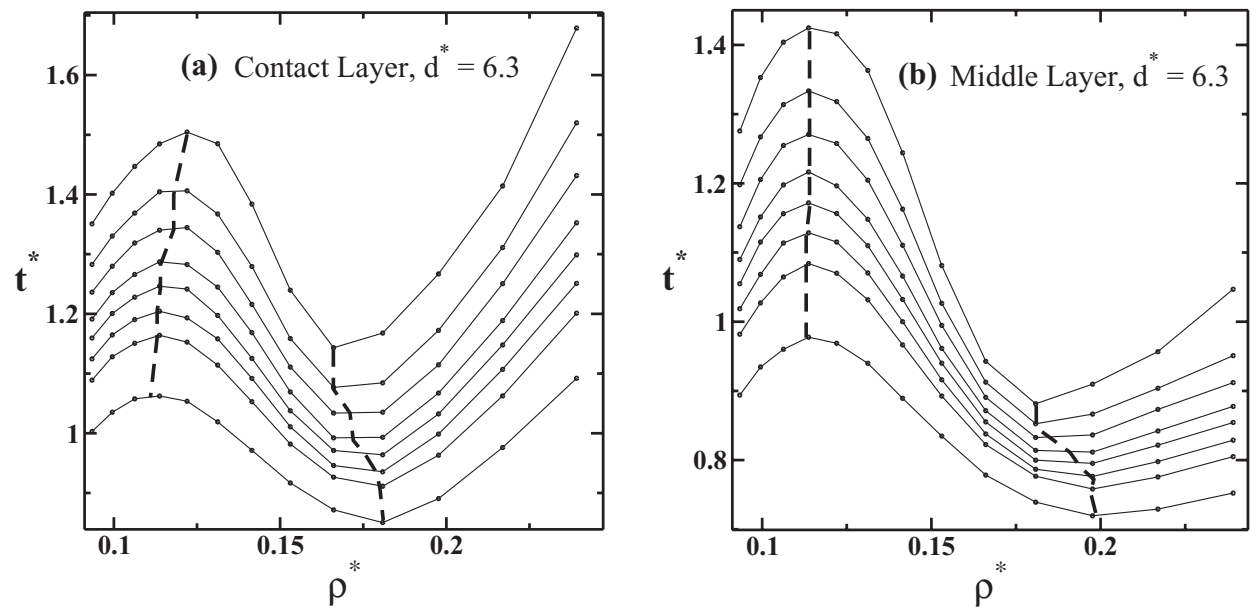

FIG. 5. Translational order parameter versus density for $d^{*}=6.3$ for (a) contact layer and (b) middle layer, separately. The solid lines represent the isotherms $T^{*}=0.170,0.190,0.205,0.220,0.232,0.245,0.260$, and 0.300 from the top to the bottom. The dashed lines connect the extremes.

The translational order parameter for the contact layer for very low temperatures and very high densities is larger than the value for the middle layer what suggests that becomes very large the indicating of crystallization of the particles at the wall. For the confinement with $d^{*}=5.5$ and 6.0, a similar behavior for $t^{*}$ is observed. The structural differences between the layers present in our model were observed not only in water but also in colloidal suspension for several kinds of particle-plates interactions $s^{36,101-104}$ and for confined SPC/E water by hydrophobic wall. ${ }^{42}$

Another property that exhibits anomalies in the bulk is the diffusion coefficient. In normal liquids, the diffusion at constant temperature grows with decreasing density, but in waterlike liquids there is a region $\left(\rho_{D_{\|} \text {-min }}^{*}<\rho^{*}<\rho_{D_{\|} \text {max }}^{*}\right)$ where the diffusion decreases with decreasing density, so this is an anomalous behavior. In our system, this anomaly can be observed in the bulk. ${ }^{78}$ How the confinement affects the region in the pressure-temperature phase diagram where the diffusion is anomalous? In order to answer this question the lateral diffusion coefficient was computed as function of den- sity as shown in Fig. 6 for (a) $d^{*}=5.5$, (b) $d^{*}=6.0$, and (c) $d^{*}=6.3$. In these cases, the diffusion coefficient has a region in which it grows with density representing the density anomalous region. The temperature of maximum and minimum diffusion coefficient is lower in the confined system than in the bulk case. ${ }^{79}$ Our findings are in agreement with the observations of the diffusion coefficient in coarse-grained model confined between smooth hydrophobic plates, separated at 0.5 $\mathrm{nm}^{51}$ and atomistic models. ${ }^{36}$

In the bulk, our potential exhibits a density anomalous region in the pressure-temperature phase diagram. How confining affects the temperature of maximum density (TMD) line? In order to answer this question, the TMD is computed under confinement. In the bulk system the TMD arises from computing $(\partial P / \partial T)_{\rho}$. As explained in Sec. III, the parallel pressure is the thermodynamic quantity related to the experimental pressure, so the density anomaly can be found through $\left(\partial P_{\|} / \partial T\right)_{\rho}$ $=0$. Fig. 7 illustrates the parallel pressure versus temperature phase diagram for (a) $d^{*}=5.5$, (b) $d^{*}=6.0$, and (c) $d^{*}$ $=6.3$ cases. The thin solid lines are the isochores, the solid
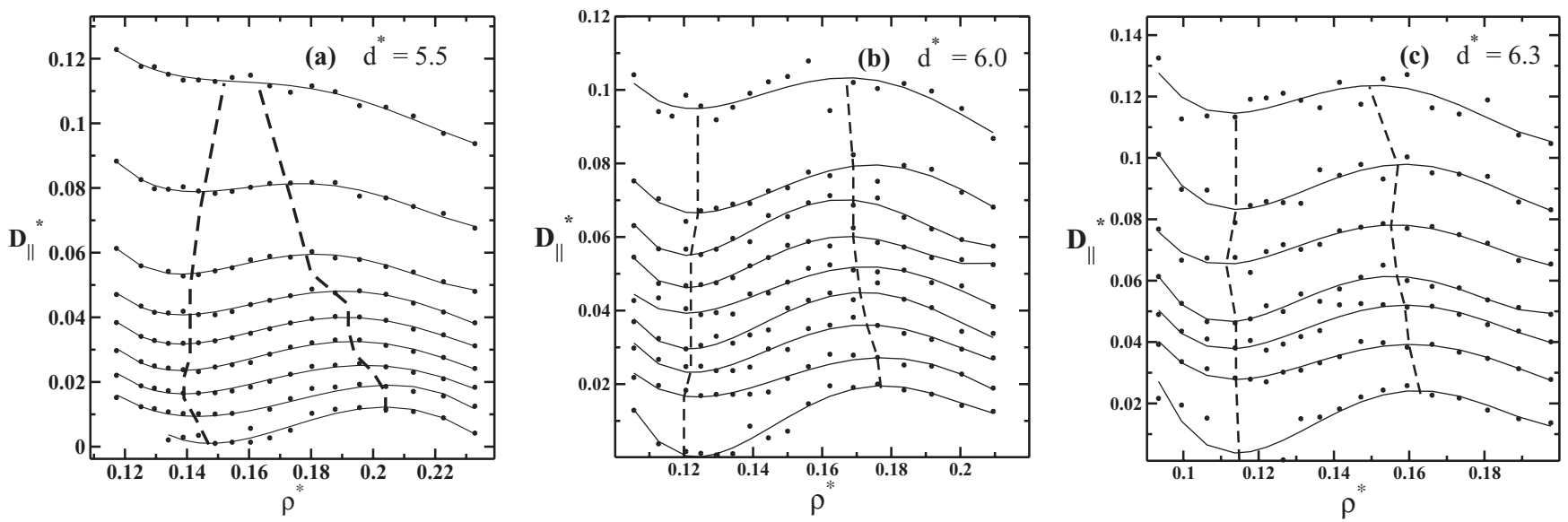

FIG. 6. Lateral diffusion coefficient as function of density for (a) $d^{*}=5.5$ and isotherms $T^{*}=0.140,0.160,0.175,0.190,0.205,0.220,0.240,0.275$, and 0.320 , (b) $d^{*}=6.0$ and isotherms $T^{*}=0.140,0.160,0.175,0.190,0.205,0.220,0.235,0.250$, and 0.290 , and (c) $d^{*}=6.3$ and isotherms $T^{*}=0.140,0.170$, $0.190,0.205,0.232,0.260$, and 0.300 , from the bottom to the top. The dots represent the simulation data and the solid line is just a polynomial fit to isotherms. The dashed lines connect the extremes of diffusion. 

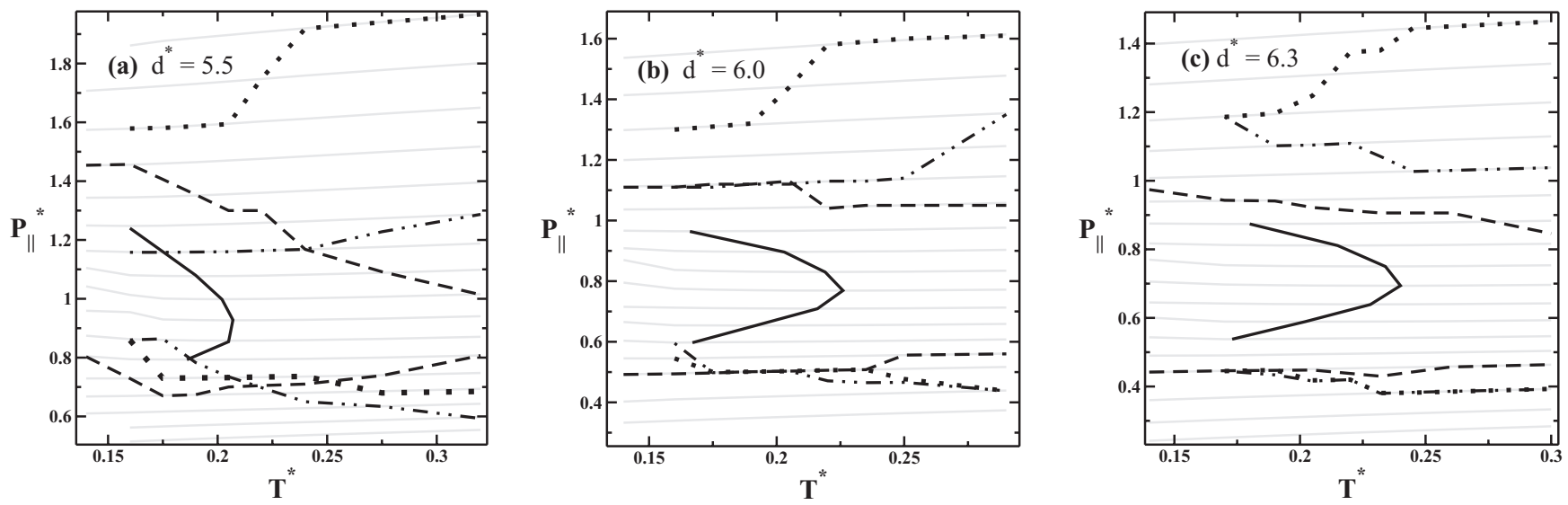

FIG. 7. Phase diagrams $P_{\|}^{*}-T^{*}$ for (a) $d^{*}=5.5$, (b) $d^{*}=6.0$, and (c) $d^{*}=6.3$. The thin solid lines are the isochores (a) $0.117 \leq \rho^{*} \leq 0.233$, (b) $0.105 \leq \rho^{*}$ $\leq 0.209$, and (c) $0.099 \leq \rho^{*} \leq 0.253$. The solid bold line represents the TMD, the dashed lines are the lateral diffusion extremes, the dashed-dotted lines are the translational order parameter extremes for a contact layer, and the dotted lines are the translational order parameter extremes for the middle layer.

bold lines represent the TMD, the dashed lines are the lateral diffusion extremes, the dashed-dotted lines are the translational order parameter extremes for a contact layer, and the dotted lines are the translational order parameter extremes for the middle layer.

A comparison between the TMD of the confined and the bulk systems is given by Fig. 8(a). The density and pressure ranges of the TMD's locations are also shown in Table I. The TMD lines of confined systems are shifted to lower temperatures and higher densities when compared with the bulk.

For $d^{*}=5.5$ and 6.0, the TMD lines are shifted to higher pressures, whereas for $d^{*}=6.3$ this shifting occurs to slightly lower pressures when compared with the bulk TMD. The nonmonotonic shift in pressure when compared with the bulk results can be attributed to the fact that we employ the lateral pressure for the confined system while we use the total pressure for the bulk system.

Kumar et al. ${ }^{36}$ found that the TMD line for confined systems is shifted to lower temperatures but in the same range of pressures when compared with the bulk system . For TIP4P water model in contact with six hydrophobic spheres, Gallo
TABLE I. Density and pressure ranges of TMD's location of confined and bulk systems.

\begin{tabular}{lcc}
\hline \hline$d^{*}$ & Density range & Pressure range \\
\hline 5.5 & $0.149<\rho^{*}<0.188$ & $0.800<P_{\|}^{*}<1.237$ \\
6.0 & $0.129<\rho^{*}<0.162$ & $0.599<P_{\|}^{*}<0.965$ \\
6.3 & $0.122<\rho^{*}<0.153$ & $0.541<P_{\|}^{*}<0.875$ \\
Bulk & $0.110<\rho^{*}<0.140$ & $0.552<P^{*}<0.913$ \\
\hline \hline
\end{tabular}

and Rovere ${ }^{23}$ found that TMD line in confined systems is shifted to lower temperatures and higher pressures. Furthermore, they observed that the spinodal curve follows the shifting of the TMD line. Similar result is observed in our systems. $\mathrm{Xu}$ and Molinero, ${ }^{105}$ using a coarse-grained model for water (mW, Monatomic Water Model) ${ }^{106}$ confined in nanopores of diameter $1.5 \mathrm{~nm}$, also found a TMD shifted to lower temperatures and higher pressures. So, the density anomalies observed in our systems have a good agreement with other atomistic and coarse-grained simulations.

The perpendicular pressure is shown in Fig. 9 as function of (a) temperature and (b) density, at $d^{*}=6.3$. For clarity,
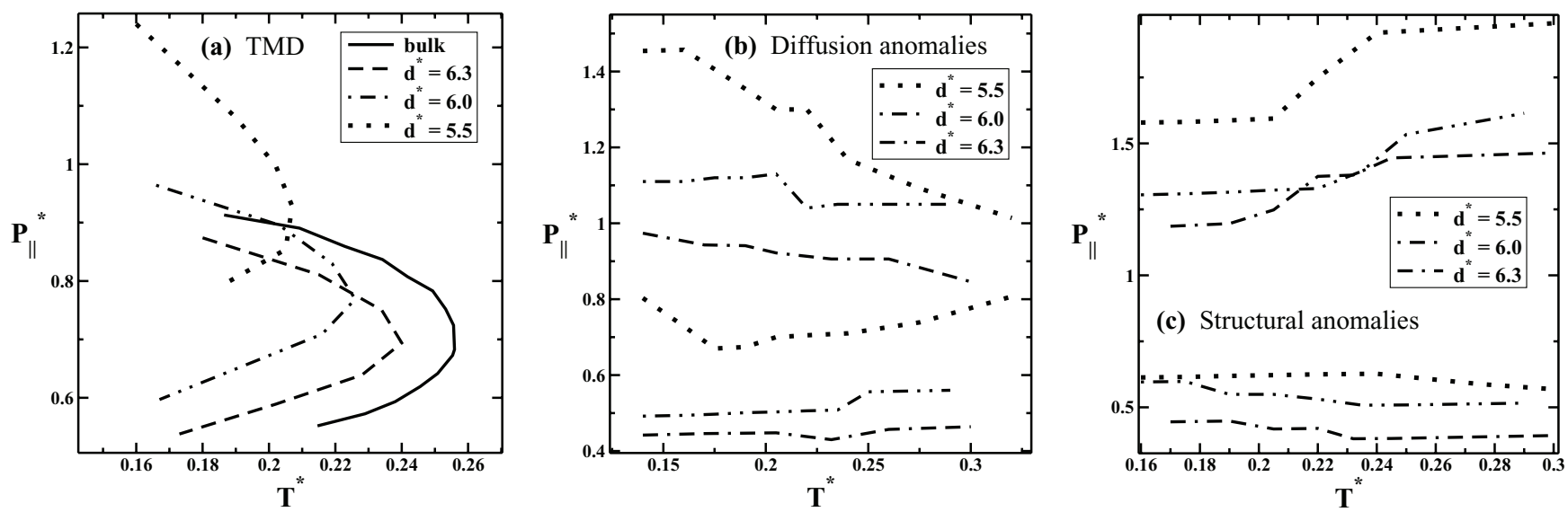

FIG. 8. Phase diagram $P_{\|}^{*}-T^{*}$ comparing in (a) the shifting of the TMD lines for the confined systems in relation to the bulk. A comparison between the anomalies of the confined systems is given in (b) for diffusion and in (c) for translational order parameter of the middle layer. For (b) and (c), the solid bold lines represent the system with $d^{*}=5.5$, the dashed-dotted lines represent $d^{*}=6.0$ and the thin dotted lines represent $d^{*}=6.3$. 

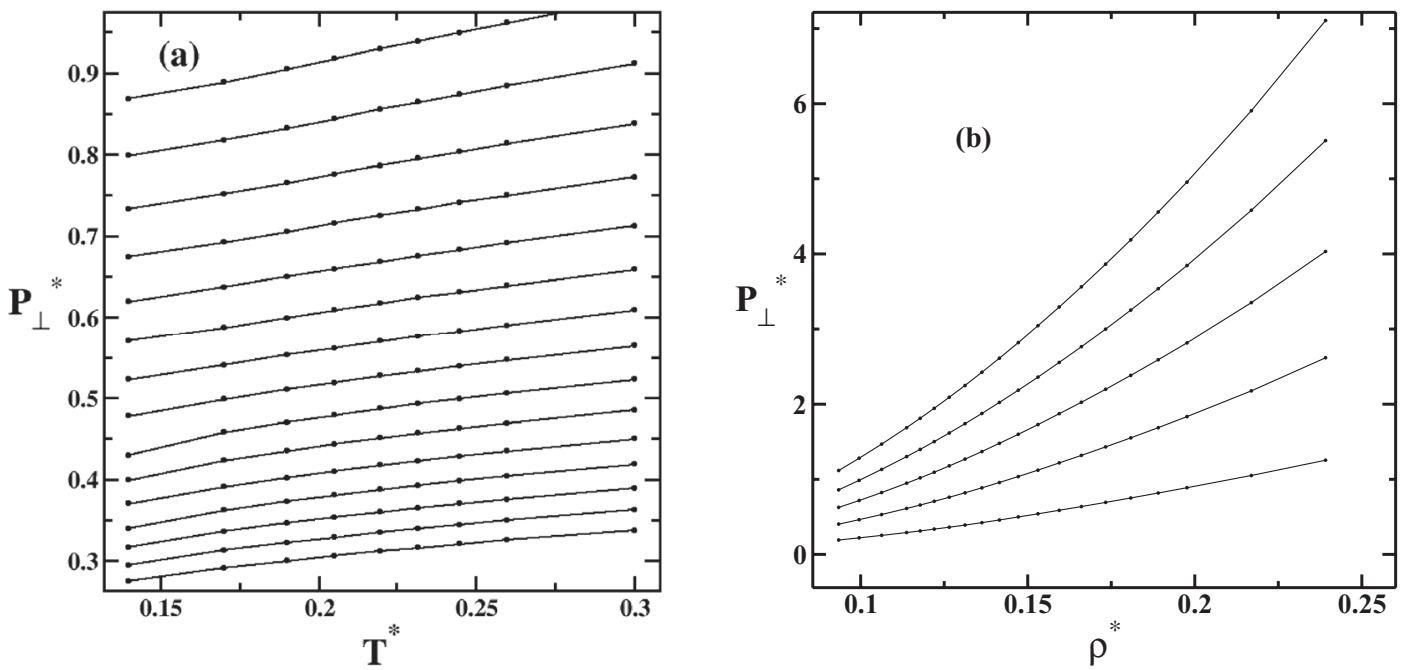

FIG. 9. Phase diagrams (a) $P_{\perp}^{*}-T^{*}$ and (b) $P_{\perp}^{*}-\rho^{*}$ at $d^{*}=6.3$. The dots are the simulation data and the solid line just connect the isochores in (a) and the isotherms in (b). The ranges of densities and temperatures are the same as shown in $P_{\|}^{*}-T^{*}$ phase diagram.

Fig. 9(b) is shifted with $n \times P_{\perp}^{*}$ where $n=1-5$ for $T^{*}$ $=0.170,0.205,0.232,0.260,0.300$, respectively. A monotonic increasing behavior is observed in both cases. The ranges of densities and temperatures are the same as shown in $P_{\|}^{*}-T^{*}$ phase diagram. The other systems $\left(d^{*}=5.5\right.$ and 6.0) have a similar behavior and they are not shown here for simplicity. The same behavior for perpendicular pressure was also observed by Kumar et al. ${ }^{36}$

In Fig. 7 we show that in the pressure-temperature phase diagram the region where the dynamic anomaly occurs englobes the region where the thermodynamic anomaly is present. This hierarchy between the anomalies is observed in a number of models ${ }^{81,100,107}$ for the bulk system and in unconfined water. ${ }^{3}$

\section{B. System with two layers}

The confinement by very narrow distances induces the transition from three to two layers. In this subsection, we study a system with plates separated by $d^{*}=4.2$. A snapshot in Fig. 10(a) shows the two contact layers, without mid- dle layer. Fig. 10(b) illustrates the behavior of the transversal density profile for fixed temperature, $T^{*}=0.220$, but for different total densities. Fig. 10(b) also shows the density profile but for fixed total density, $\rho^{*}=0.155$, and several temperatures. The structuring in just two contact layers is due the strong effect of confinement. Structure of bilayer is observed for hydrophobic confinement in TIP5P, ${ }^{108-110}$ TIP4P, ${ }^{111}$ and $\mathrm{mW}^{112}$ models of water.

Figure 11(a) shows the parallel pressure versus temperature phase diagram. The isochores are represented by thin lines, and the TMD by the solid bold line. It also shows the diffusion extrema (dashed line) and the translational order parameter extrema (dashed-dotted line). The comparison between the TMD line for the $d^{*}=4.2$ case and the TMD for the bulk system is illustrated in Fig. 11(b). The location of the TMD is $0.604<P_{\|}^{*}<0.959$ and $0.137<\rho^{*}<0.170$. Similar to what happens in the three layer system, the TMD line shifts to lower temperatures when compared with the bulk. This characteristic is evidenced in Fig. 11(c).

Figure 12(a) illustrates the perpendicular pressure versus temperature phase diagram for various isochores that show (a)

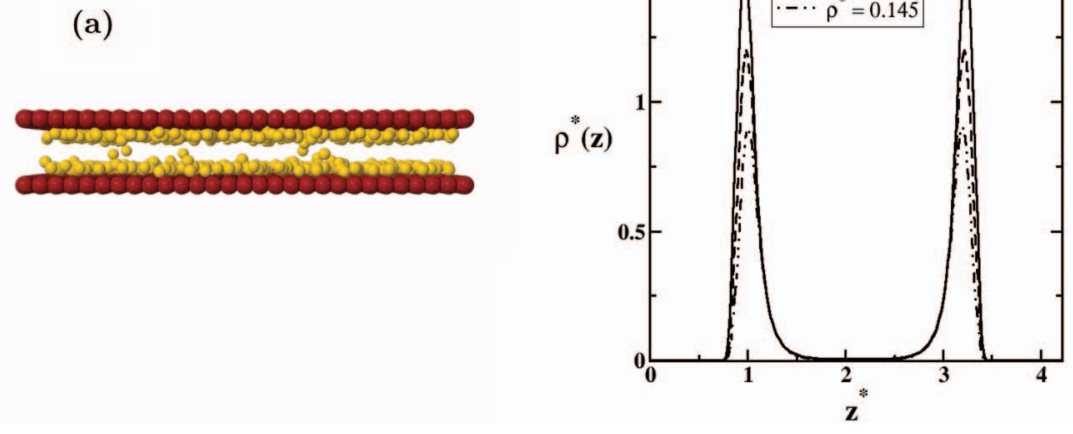

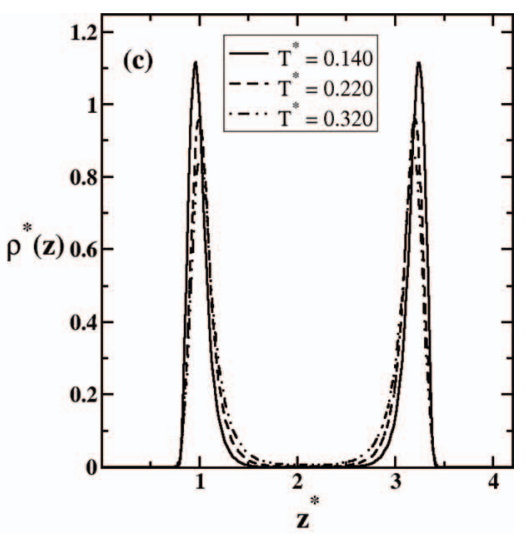

FIG. 10. (a) Snapshot of the system after the equilibrium state. In (b) we can see the transversal density profile for $T^{*}=0.220$ and several densities, and in (c) for $\rho^{*}=0.155$ and several temperatures. 

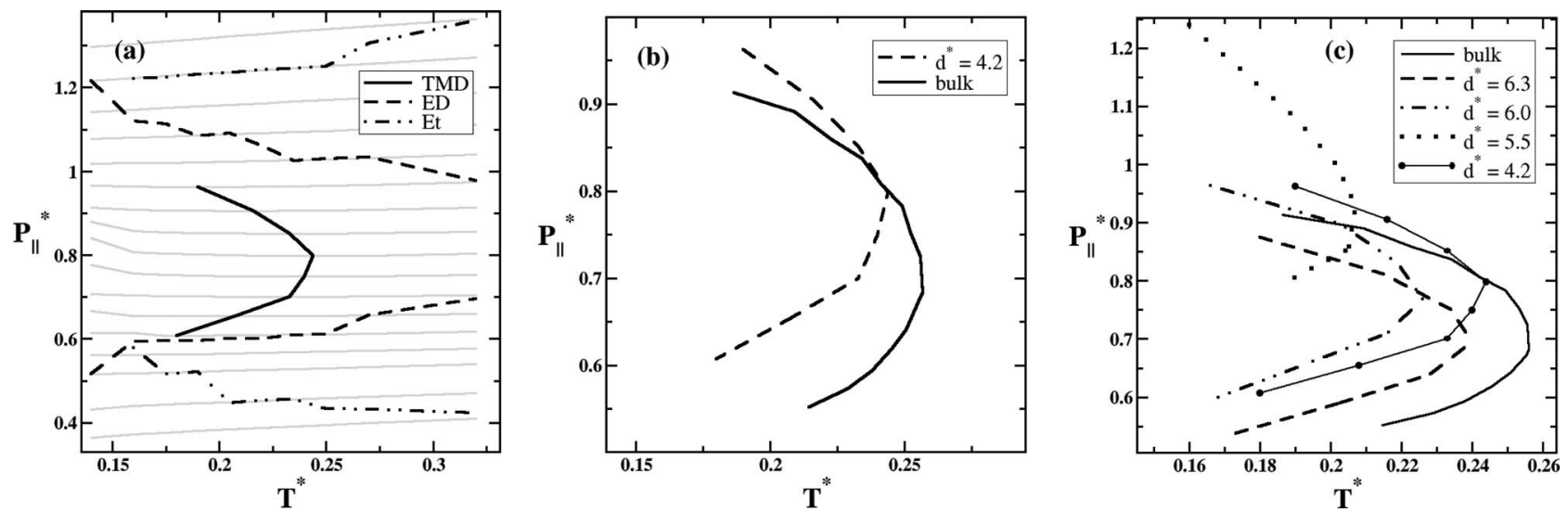

FIG. 11. Phase diagrams $P_{\|}^{*}-T^{*}$ showing the density, dynamic, and structural anomalies in (a). In (b) we have a comparison between the TMD lines of $d^{*}=$ 4.2 and the bulk system, and in (c) the same comparison is done with TMD lines for systems that present formation of three layers.

no TMD line. No anomalous behavior is observed similar to what happens in the three layers regime. Figure 12 also shows the radial distribution function (in (b)) and the mean square displacement (in (c)) for the lateral direction. For $\rho^{*}=0.137$, these figures show a amorphous solidlike behavior for $T^{*}=$ 0.160 and a liquidlike behavior for $T^{*}=0.270$. A solid-toliquid transition was also observed for the TIP5P model, ${ }^{109,110}$ for the TIP4P model, ${ }^{111}$ and for the mW model. ${ }^{112}$

An anomalous region for translational order parameter and for lateral diffusion are also observed in this extremely confined system as can be seen in Figs. 13(a) and 13(b), respectively. The dashed lines connect the extremes of these anomalies, defining the anomalous region that we can see in $P_{\|}^{*}-T^{*}$ phase diagram in Fig. 11(a).

\section{Three-to-two layers system}

The system with $d^{*}=4.8$ exhibits an unusual behavior that resembles properties of both two layers and the three layers systems. In Fig. 14(a) the lateral diffusion as function of density is illustrated. The diffusion anomaly is only present at very low temperatures, $T^{*} \leq 0.150$. In addition, as shown in Fig. 14(b) the TMD line is also restricted to low tempera- tures. In Fig. 14(c) the comparison between the TMD line of the confined system with $d^{*}=4.8$ and the TMD of bulk confirms this shift of the TMD to very low $T^{*}$, much lower than the shift observed for confinement with $4.8<d^{*}$ and $4.8>$ $d^{*}$ discussed above.

This $d^{*}=4.8$ case is not only peculiar for exhibiting a very low temperature of maximum density but also for presenting anomalous behavior at the perpendicular pressure versus temperature phase diagram. In Fig. 15(a) the isochores at the perpendicular pressure versus temperature phase diagram exhibit a minimum that is only present in the case $d^{*}=4.8$ but not in the previous analyzed cases where $d^{*}$ $>4.8$ or $d^{*}<4.8$. In order to shade some light on the reason for the unusual behavior of the system for $d^{*}=4.8$ we explore the behavior of the structure and of the stability of the layers. Fig. 15(b) shows the lateral pressure versus density of fixed temperatures. For $T^{*}<0.190$, the slope of the curve first increases and then decreases. This change even though not indicating a phase transition is usually observed before the phase separation is established. ${ }^{48}$

Figure 15(c) shows the transversal density profile for $\rho^{*}$ $=0.139$ and for a number of temperatures. At this density, three layers are present for low temperatures, $T^{*}=0.118$
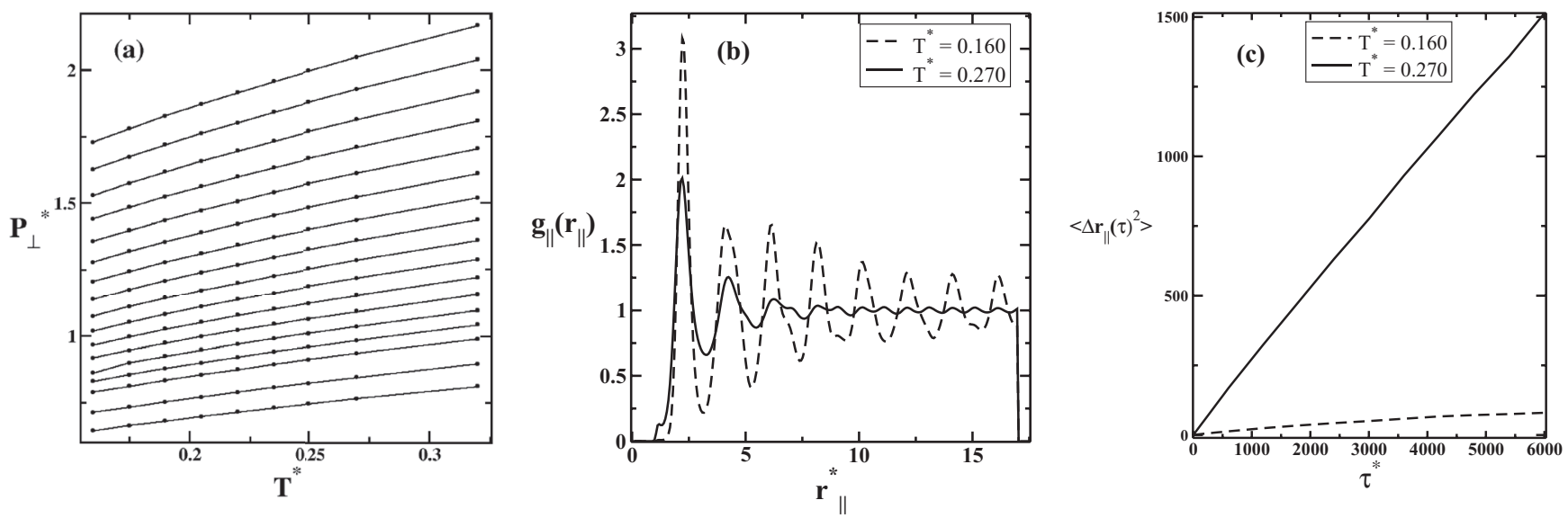

FIG. 12. In (a), the monotonic increasing behavior is observed for perpendicular pressure with the temperature. In (b), we have a $g_{\|}\left(r_{\|}\right)$for a amorphous solidlike state, with $T^{*}=0.160$, and a liquidlike state, with $T^{*}=0.270$, both at $\rho^{*}=0.137$. The MSD is observed for these cases in (c). 

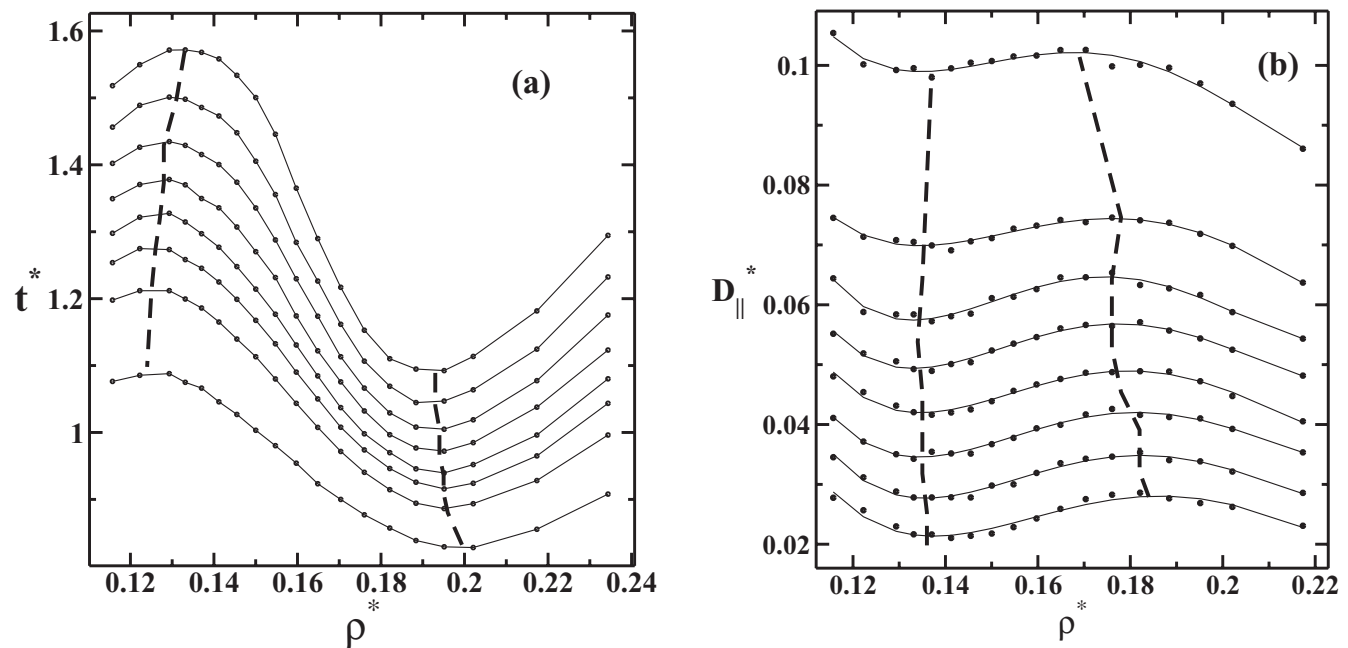

FIG. 13. In (a), the translational order parameter as function of density, and in (b) the lateral diffusion coefficient as function of density. The isotherms connect the simulation data (points) and they are from the top to the bottom $T^{*}=0.175,0.190,0.205,0.220,0.235,0.250,0.270$, and 0.320 for $t^{*}$ (a) and from the bottom to the top for $D_{\|}^{*}$ (b). The dashed lines connect the extremes observed in both cases.
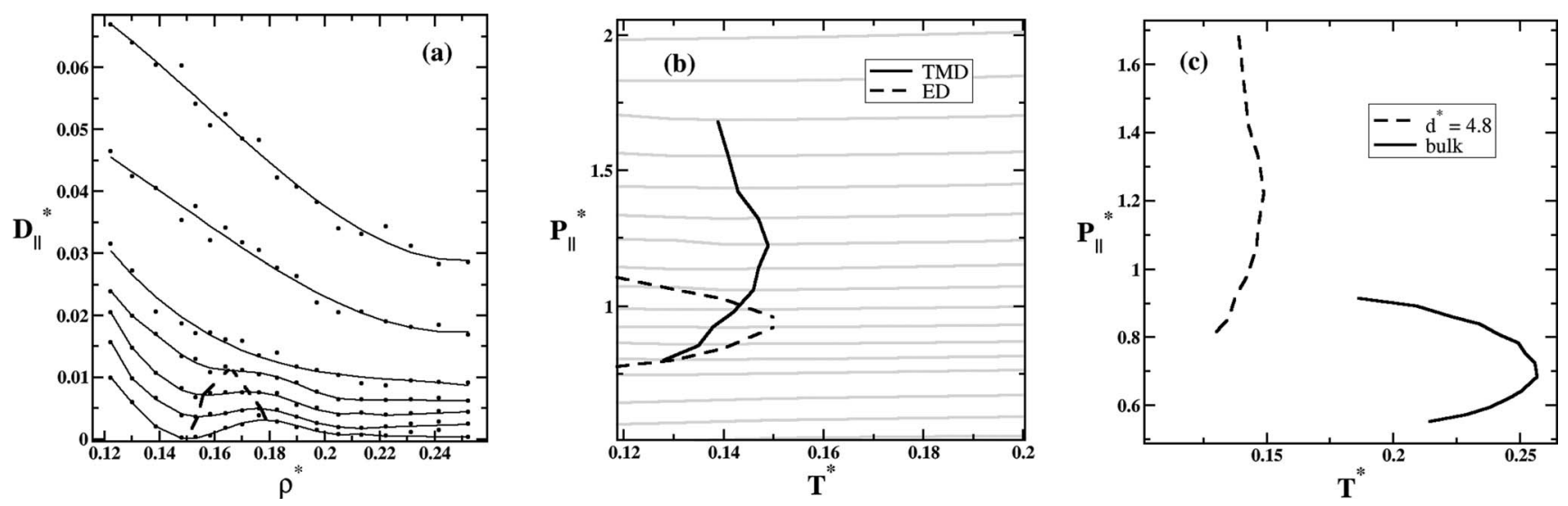

FIG. 14. In (a), the lateral diffusion coefficient as function of density for isotherms from the bottom to the top $T^{*}=0.118,0.130,0.140,0.150,0.160,0.190$, and 0.220. Diffusion extremes are connected by dashed lines. In (b), we have the phase diagram showing the TMD line and the diffusion extremes. A comparison between the TMD line of this system with bulk is given in (c).
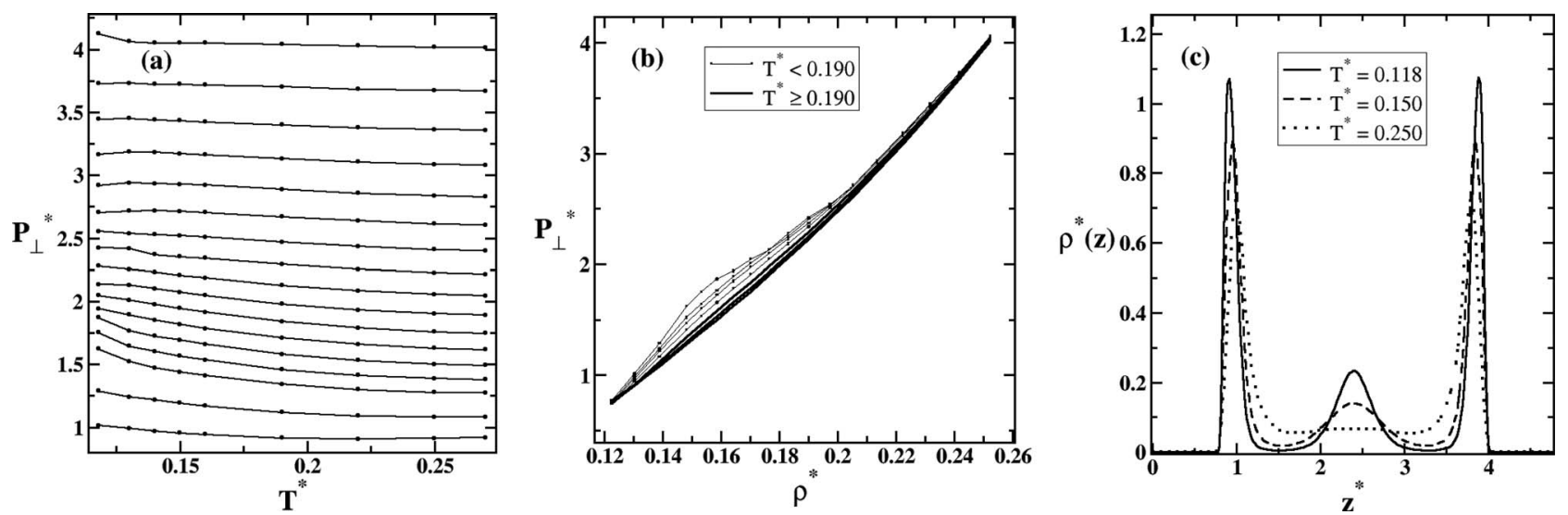

FIG. 15. The perpendicular pressure versus temperature is given in (a) and versus density is given in (b). The simulation data (dots) are connected by solid lines for better visualization. The transversal density profile for $\rho^{*}=0.139$ and some temperatures are shown in (c). 

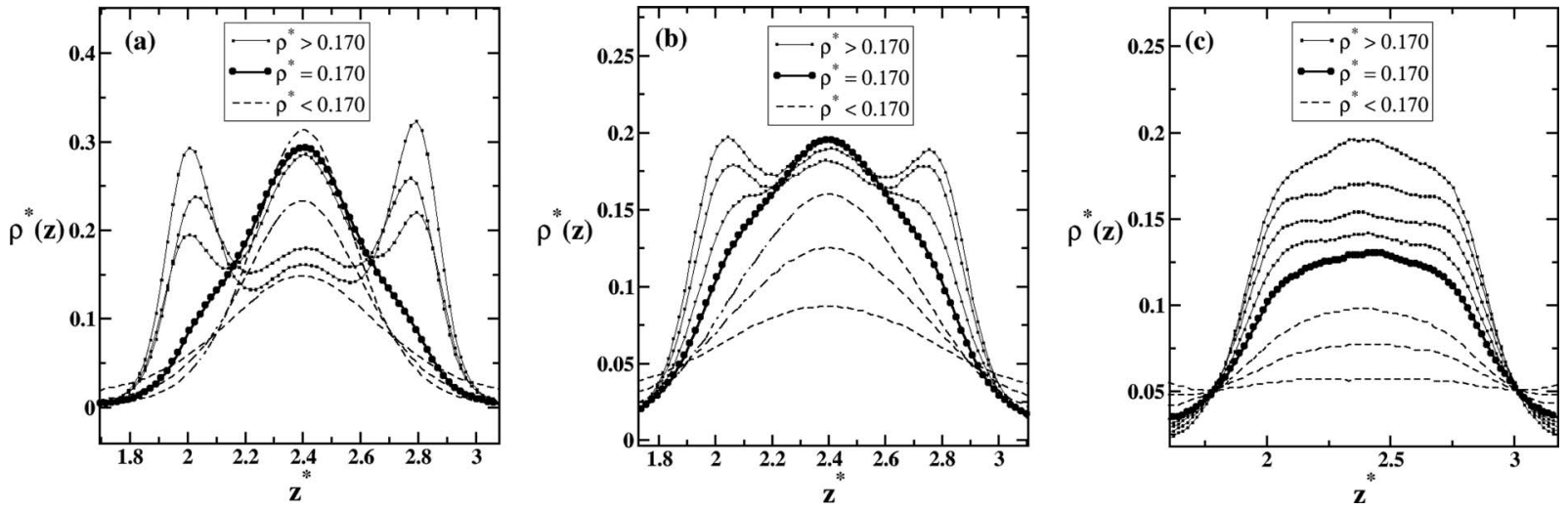

FIG. 16. Transversal density profile for the middle layer at many densities and (a) $T^{*}=0.118$, (b) $T^{*}=0.160$, and (c) $T^{*}=0.220$. The dashed lines represent states for $\rho^{*}<0.170$, the solid lines for $\rho^{*}>0.170$, and the bold circles are for $\rho^{*}=0.170$.

and 0.150. At high temperatures, $T^{*}=0.250$, two layers are well defined with particles equally distributed between them without forming a third layer.

In order to understand the two-to-three layers transition, the change in the structure of the central layer with temperature and density is checked. The structure of the middle layer is shown in Fig. 16 for different temperatures and densities. At low temperatures (cases (a) at $T^{*}=0.118$ and (b) at $T^{*}=$ 0.160 ) and high densities, $\rho^{*}>0.170$, the central layer is divided in many sublayers. By decreasing the density the many layers give rise at $\rho^{*}=0.170$ to a central layer that disappears as the density is decreased any further. At high temperatures, $T^{*}>0.160$ (case (c) shown at $T^{*}=0.220$ ), as the density decreases the system passes from three-to-two layers without forming the sublayers. At low temperatures, due to the presence of the many sublayers the density anomalous region appears. The TMD originates from particles moving from one scale to the other. ${ }^{79}$

Our interpretation is in accordance with the assumption of Kumar et al. ${ }^{36}$ that the split of the middle layer in sublayers is justified by the density anomaly. Using SPC/E model for water and hydrophobic rough plates, Giovambattista et al. ${ }^{42}$ found a phase transition between a bilayer ice and a trilayer heterogeneous fluid for different distances between the plates. For smooth plates separated at $0.8 \mathrm{~nm}$ and SPC/E model, Lombardo et al. ${ }^{49}$ also found a phase transition between two and three layers.

Changes in diffusion coefficient as function of separation between the plates are reported in systems with transitions between 6 and 5, 5 and 4, and 4 and 3 layers, ${ }^{113}$ suggesting that the dynamic behavior changes in systems with structural transitions. So, the dynamic behavior of our system at $d^{*}=$ 4.8 is another possible explanation for the peculiar behavior on its anomalies.

\section{CONCLUSIONS}

In this paper, the effects of confining a system of particles interacting through core-softened is explored.

The formation of three layers, two close to the walls and one central, is observed for large values of $d^{*}$, while two layers are observed for small values of $d^{*}$. In addition the region in the pressure-temperature phase diagram where the density anomaly appears moves to lower temperatures. These results are similar to the results obtained in atomistic and coarsegrained models where unlike our model the directionality of the h-bonds is explicitly included. ${ }^{36,114}$

Our results indicate that layers are formed in order to minimize the particle-particle interaction potential and the wall-particle interaction. Therefore, if the walls are distant at $d^{*}=5.0$ it is possible to fit three layers in which each is distant at 2.0 from the other and the contact layer is distant at 1.0 from the wall. For $d^{*}=4.0$ it is only possible to fit two layers distant at 2.0 from each other and 1.0 from the wall. The density, diffusion, and structural anomalous behavior that implies particles moving from one length scales to the other (moving from the length scale at $\approx 3.0$ to the length scales at 1.0 ) occurs only along the parallel plane, therefore the anomalies appear as function of $P_{\|}$.

The case $d^{*}=4.8$ is the boundary between the two layer and the three layer cases. This case allows us to observe how the presence of the density anomaly is related with moving from different particle-particle distances.

In all the confined cases we show that in the pressuretemperature phase diagram the region where the dynamic anomaly occurs englobes the region where the thermodynamic anomaly is present which is also observed in the bulk system. Therefore, the confinement does not disturb this hierarchy.

Our results suggest that effective spherical symmetric two length scales potentials are an interesting tool for understanding the mechanisms that arise from confining systems with density, diffusion, and structural anomalies. Due to their simplicity the results obtained can be generalized to other experimental realizations besides water.

\section{ACKNOWLEDGMENTS}

We thank for financial support given by the Brazilian science agencies, CNPq, and Capes. This work is partially supported by CNPq and INCT-FCx. We also thank CEFIC - Centro de Física Computacional of Physics Institute at UFRGS for the computer clusters. 
${ }^{1}$ R. Waler, Essays of Natural Experiments (Johnson Reprint, New York, 1964).

${ }^{2}$ G. S. Kell, J. Chem. Eng. Data 20, 97 (1975).

${ }^{3}$ C. A. Angell, E. D. Finch, L. A. Woolf, and P. Bach, J. Chem. Phys. 65, 3063 (1976).

${ }^{4}$ R. J. Speedy and C. A. Angell, J. Chem. Phys. 65, 851 (1976).

${ }^{5}$ H. Kanno and C. A. Angell, J. Chem. Phys. 70, 4008 (1979).

${ }^{6}$ C. A. Angell, M. Oguni, and W. J. Sichina, J. Chem. Phys. 86, 998 (1982).

${ }^{7}$ E. Tombari, C. Ferrari, and G. Salvetti, Chem. Phys. Lett. 300, 749 (1999).

${ }^{8}$ F. X. Prielmeier, E. W. Lang, R. J. Speedy, and H.-D. Lüdemann, Phys. Rev. Lett. 59, 1128 (1987).

${ }^{9}$ P. H. Poole, F. Sciortino, U. Essmann, and H. E. Stanley, Nature (London) 360, 324 (1992).

${ }^{10}$ Y. Liu, A. Z. Panagiotopoulos, and P. G. Debenedetti, J. Chem. Phys. 131, 104508 (2009).

${ }^{11}$ F. Sciortino, I. Saika-Voivod, and P. H. Poole, Phys. Chem. Chem. Phys. 13, 19759 (2011).

${ }^{12}$ T. A. Kesselring, G. Franzese, S. V. Buldyrev, H. J. Herrmann, and H. E. Stanley, Sci. Rep. 2, 474 (2012).

${ }^{13}$ L. Liu, S.-H. Chen, A. Faraone, S.-W. Yen, and C.-Y. Mou, Phys. Rev. Lett. 95, 117802 (2005).

${ }^{14}$ L. Xu, P. Kumar, S. V. Buldyrev, S.-H. Chen, P. Poole, F. Sciortino, and H. E. Stanley, Proc. Natl. Acad. Sci. U.S.A. 102, 16558 (2005).

${ }^{15}$ M. Erko, G. H. Findenegg, N. Cade, A. G. Michette, and O. Paris, Phys. Rev. B 84, 104205 (2011).

${ }^{16}$ K. Morishige and K. Nobuoka, J. Chem. Phys. 107, 6965 (1997).

${ }^{17}$ K. Morishige and K. Kawano, J. Chem. Phys. 110, 4867 (1999).

${ }^{18}$ K. Koga, G. T. Gao, H. Tanaka, and X. C. Zeng, Nature (London) 412, $802(2001)$

${ }^{19}$ G. Hummer, J. C. Rasaiah, and J. P. Noworyta, Nature (London) 414, 188 (2001).

${ }^{20}$ J. R. Bordin, A. B. de Oliveira, A. Diehl, and M. C. Barbosa, J. Chem. Phys. 137, 084504 (2012).

${ }^{21}$ J. R. Bordin, A. Diehl, M. C. Barbosa, and Y. Levin, Phys. Rev. E 85, 031914 (2012).

${ }^{22}$ D. Corradini, P. Gallo, and M. Rovere, J. Mol. Liq. 159, 18 (2011).

${ }^{23}$ P. Gallo and M. Rovere, Phys. Rev. E 76, 061202 (2007).

${ }^{24}$ P. Gallo, M. Rapinesi, and M. Rovere, J. Chem. Phys. 117, 369 (2002).

${ }^{25}$ P. Gallo, M. Rovere, and S.-H. Chen, J. Phys. Chem. Lett. 1, 729 (2010).

${ }^{26}$ P. Gallo, M. Rovere, and S.-H. Chen, J. Phys.: Condens. Matter 24, 064109 (2012).

${ }^{27}$ F. Mallamace, M. Broccio, C. Corsaro, A. Faraone, L. Liu, C.-Y. Mou, and S.-H. Chen, J. Phys.: Condens. Matter 18, S2285 (2006).

${ }^{28}$ A. Faraone, K.-H. Liu, C.-Y. Mou, Y. Zhang, and S.-H. Chen, J. Chem. Phys. 130, 134512 (2009).

${ }^{29}$ E. G. Strekalova, M. G. Mazza, H. E. Stanley, and G. Franzese, J. Phys.: Condens. Matter 24, 064111 (2012).

${ }^{30}$ E. G. Strekalova, J. Luo, H. E. Stanley, G. Franzese, and S. V. Buldyrev, Phys. Rev. Lett. 109, 105701 (2012).

${ }^{31}$ P. A. Bonnaud, B. Coasne, and R. J.-M. Pellenq, J. Phys.: Condens. Matter 22, 284110 (2010).

${ }^{32}$ O. Pizio, H. Dominguez, L. Pusztai, and S. Sokolowski, Physica A 388, 2278 (2009).

${ }^{33}$ M. Bellissent-Funel, R. SridiDorbez, and L. Bosio, J. Chem. Phys. 104, 10023 (1996).

${ }^{34}$ J.-M. Zanotti, M.-C. Bellissent-Funel, and S.-H. Chen, Europhys. Lett. 71, 91 (2005).

${ }^{35}$ K. Koga and H. Tanaka, J. Chem. Phys. 122, 104711 (2005).

${ }^{36}$ P. Kumar, S. V. Buldyrev, F. W. Starr, N. Giovambattista, and H. E. Stanley, Phys. Rev. E 72, 051503 (2005).

${ }^{37}$ M. Lupkowski and F. van Swol, J. Chem. Phys. 93, 737 (1990).

${ }^{38}$ P. Scheidler, W. Kob, and K. Binder, Europhys. Lett. 59, 701 (2002).

${ }^{39}$ N. Choudhury, J. Chem. Phys. 132, 064505 (2010).

${ }^{40}$ X.-Q. Chu, K.-H. Liu, M. S. Tyagi, C.-Y. Mou, and S.-H. Chen, Phys. Rev. E 82, 020501 (2010).

${ }^{41}$ M. Meyer and H. E. Stanley, J. Phys. Chem. B 103, 9728 (1999).

${ }^{42}$ N. Giovambattista, P. J. Rossky, and P. G. Debenedetti, Phys. Rev. Lett. 102, 050603 (2009).

${ }^{43}$ J. Deschamps, F. Audonnet, N. Brodie-Linder, M. Schoeffel, and C. AlbaSimionesco, Phys. Chem. Chem. Phys. 12, 1440 (2010).

${ }^{44}$ S. Jähnert, F. V. Chávez, G. E. Schaumann, A. Schreiber, M. Schönhoff, and G. H. Findenegg, Phys. Chem. Chem. Phys. 10, 6039 (2008).

${ }^{45}$ M.-C. Bellissent-Funel, J. Lal, and L. Bosio, J. Chem. Phys. 98, 4246 (1993).
${ }^{46}$ D. W. Hwang, C.-C. Chu, A. K. Sinha, and L.-P. Hwang, J. Chem. Phys. 126, 044702 (2007)

${ }^{47}$ S. Kittaka, K. Sou, T. Yamaguchi, and K. Tozaki, Phys. Chem. Chem. Phys. 11, 8538 (2009).

${ }^{48}$ N. Giovambattista, P. J. Rossky, and P. G. Debenedetti, J. Phys. Chem. B 113, 13723 (2009).

${ }^{49}$ T. G. Lombardo, P. J. Rossky, and P. G. Debenedetti, Faraday Discuss. 141, 359 (2009).

${ }^{50}$ N. Giovambattista, P. J. Rossky, and P. G. Debenedetti, Phys. Rev. E 73, 041604 (2006).

${ }^{51}$ F. Santos and G. Franzese, J. Phys. Chem. B 115, 14311 (2011).

${ }^{52} \mathrm{H}$. Thurn and J. Ruska, J. Non-Cryst. Solids 22, 331 (1976).

${ }^{53}$ G. E. Sauer and L. B. Borst, Science 158, 1567 (1967).

${ }^{54}$ S. J. Kennedy and J. C. Wheeler, J. Chem. Phys. 78, 1523 (1983).

${ }^{55}$ T. Tsuchiya, J. Phys. Soc. Jpn. 60, 227 (1991).

${ }^{56}$ P. T. Cummings and G. Stell, Mol. Phys. 43, 1267 (1981).

${ }^{57}$ M. Togaya, Phys. Rev. Lett. 79, 2474 (1997).

${ }^{58}$ C. A. Angell, R. D. Bressel, M. Hemmatti, E. J. Sare, and J. C. Tucker, Phys. Chem. Chem. Phys. 2, 1559 (2000).

${ }^{59}$ R. Sharma, S. N. Chakraborty, and C. Chakravarty, J. Chem. Phys. 125, 204501 (2006).

${ }^{60}$ M. S. Shell, P. G. Debenedetti, and A. Z. Panagiotopoulos, Phys. Rev. E 66, 011202 (2002).

${ }^{61}$ S. Sastry and C. A. Angell, Nature Mater. 2, 739 (2003).

${ }^{62}$ S.-H. Chen, F. Mallamace, C.-Y. Mou, M. Broccio, C. Corsaro, A. Faraone, and L. Liu, Proc. Natl. Acad. Sci. U.S.A. 103, 12974 (2006).

${ }^{63}$ T. Morishita, Phys. Rev. E 72, 021201 (2005).

${ }^{64}$ P. C. Hemmer and G. Stell, Phys. Rev. Lett. 24, 1284 (1970).

${ }^{65}$ A. Scala, M. R. Sadr-Lahijany, N. Giovambattista, S. V. Buldyrev, and H. E. Stanley, J. Stat. Phys. 100, 97 (2000).

${ }^{66}$ S. V. Buldyrev, G. Franzese, N. Giovambattista, G. Malescio, M. R. SadrLahijany, A. Scala, A. Skibinsky, and H. E. Stanley, Physica A 304, 23 (2002).

${ }^{67}$ C. Buzano and M. Pretti, J. Chem. Phys. 119, 3791 (2003).

${ }^{68}$ A. Skibinsky, S. V. Buldyrev, G. Franzese, G. Malescio, and H. E. Stanley, Phys. Rev. E 69, 061206 (2004).

${ }^{69}$ G. Franzese, G. Malescio, A. Skibinsky, S. V. Buldyrev, and H. E. Stanley, Phys. Rev. E 66, 051206 (2002).

${ }^{70}$ A. L. Balladares and M. C. Barbosa, J. Phys.: Condens. Matter 16, 8811 (2004).

${ }^{71}$ A. B. de Oliveira and M. C. Barbosa, J. Phys.: Condens. Matter 17, 399 (2005).

${ }^{72}$ V. B. Henriques and M. C. Barbosa, Phys. Rev. E 71, 031504 (2005).

${ }^{73}$ V. B. Henriques, N. Guissoni, M. A. Barbosa, M. Thielo, and M. C. Barbosa, Mol. Phys. 103, 3001 (2005).

${ }^{74}$ E. A. Jagla, Phys. Rev. E 58, 1478 (1998)

${ }^{75}$ N. B. Wilding and J. E. Magee, Phys. Rev. E 66, 031509 (2002).

${ }^{76} \mathrm{~S}$. Maruyama, K. Wakabayashi, and M. A. Oguni, AIP Conf. Proc. 708, 675 (2004).

${ }^{77}$ R. Kurita and H. Tanaka, Science 306, 845 (2004).

${ }^{78}$ A. B. de Oliveira, P. A. Netz, T. Colla, and M. C. Barbosa, J. Chem. Phys. 124, 084505 (2006).

${ }^{79}$ A. B. de Oliveira, P. A. Netz, T. Colla, and M. C. Barbosa, J. Chem. Phys. 125, 124503 (2006).

${ }^{80}$ A. B. de Oliveira, M. C. Barbosa, and P. A. Netz, Physica A 386, 744 (2007).

${ }^{81}$ A. B. de Oliveira, P. A. Netz, and M. C. Barbosa, Eur. Phys. J. B 64, 481 (2008).

${ }^{82}$ A. B. de Oliveira, G. Franzese, P. A. Netz, and M. C. Barbosa, J. Chem. Phys. 128, 064901 (2008).

${ }^{83}$ A. B. de Oliveira, P. A. Netz, and M. C. Barbosa, Europhys. Lett. 85, 36001 (2009).

${ }^{84}$ N. V. Gribova, Y. D. Fomin, D. Frenkel, and V. N. Ryzhov, Phys. Rev. E 79, 051202 (2009).

${ }^{85}$ E. Lomba, N. G. Almarza, C. Martin, and C. McBride, J. Chem. Phys. 126, 244510 (2007).

${ }^{86}$ M. Girardi, M. Szortyka, and M. C. Barbosa, Physica A 386, 692 (2007).

${ }^{87}$ D. Y. Fomin, N. V. Gribova, V. N. Ryzhov, S. M. Stishov, and D. Frenkel, J. Chem. Phys. 129, 064512 (2008).

${ }^{88}$ N. M. Barraz, Jr., E. Salcedo, and M. C. Barbosa, J. Chem. Phys. 131, 094504 (2009).

${ }^{89}$ J. da Silva, E. Salcedo, A. B. Oliveira, and M. C. Barbosa, J. Chem. Phys. 133, 244506 (2010). 
${ }^{90}$ N. J. D. Weeks, D. Chandler, and H. C. Andersen, J. Chem. Phys. 54, 5237 (1971).

${ }^{91}$ D. Frenkel and B. Smit, Understanding Molecular Simulation, 1st ed. (Academic, San Diego, 1996).

${ }^{92}$ M. P. Allen and D. J. Tildesley, Computer Simulations of Liquids, 1 st ed. (Claredon, Oxford, 1987).

${ }^{93}$ W. G. Hoover, Phys. Rev. A 31, 1695 (1985).

${ }^{94}$ W. G. Hoover, Phys. Rev. A 34, 2499 (1986).

${ }^{95}$ P. Kumar, S. Han, and H. E. Stanley, J. Phys.: Condens. Matter 21, 504108 (2009).

${ }^{96}$ P. Kumar, F. W. Starr, S. V. Buldyrev, and H. E. Stanley, Phys. Rev. E 75, 011202 (2007)

${ }^{97}$ K. Koga, X. C. Zeng, and H. Tanaka, Chem. Phys. Lett. 285, 278 (1998).

${ }^{98}$ R. Zangi and S. A. Rice, Phys. Rev. E 61, 660 (2000).

${ }^{99}$ J. E. Errington, P. G. Debenedetti, and S. Torquato, J. Chem. Phys. 118, 2256 (2003).

${ }^{100}$ J. R. Errington and P. G. Debenedetti, Nature (London) 409, 318 (2001).
${ }^{101}$ R. Radhakrishnan, K. E. Gubbins, and M. S-Bartkowiak, J. Chem. Phys. 112, 11048 (2000).

102 J. E. Hug, F. van Swol, and C. F. Zukoski, Langmuir 11, 111 (1995).

${ }^{103}$ S. R.-V. Castrillon, N. Giovambattista, I. A. Aksay, and P. G. Debenedetti, J. Chem. Phys. B 113, 1438 (2009).

${ }^{104}$ S. Han, P. Kumar, and H. E. Stanley, Phys. Rev. E 79, 041202 (2009).

${ }^{105}$ L. Xu and V. Molinero, J. Phys. Chem. B 115, 14210 (2011).

${ }^{106}$ V. Molinero and E. B. Moore, J. Phys. Chem. B 113, 4008 (2009).

${ }^{107}$ P. A. Netz, F. W. Starr, H. E. Stanley, and M. C. Barbosa, J. Chem. Phys. 115, 344 (2001).

${ }^{108}$ R. Zangi and A. E. Mark, Phys. Rev. Lett. 91, 025502 (2003).

${ }^{109}$ R. Zangi and A. E. Mark, J. Chem. Phys. 119, 1694 (2003).

${ }^{110}$ S. Han, M. Y. Choi, P. Kumar, and H. E. Stanley, Nat. Phys. 6, 685 (2010).

${ }^{111}$ K. Koga, X. C. Zeng, and H. Tanaka, Phys. Rev. Lett. 79, 5262 (1997).

${ }^{112}$ N. Kastelowitz, J. C. Johnston, and V. Molinero, J. Chem. Phys. 132, 124511 (2010)

${ }^{113}$ J. Gao, W. D. Luedtke, and U. Landman, Phys. Rev. Lett. 79, 705 (1997).

${ }^{114}$ S. Han, P. Kumar, and H. E. Stanley, Phys. Rev. E 77, 030201 (2008). 